\title{
Classifier-free Extraction of Power Line Wires from Point Cloud Data
}

\author{
Mohammad Awrangjeb and Yongsheng Gao \\ Institute for Integrated and Intelligent Systems \\ Griffith University \\ Nathan QLD 4111 Australia \\ $\{$ m.awrangjeb, yongsheng.gao\}@griffith.edu.au
}

\author{
Guojun Lu \\ School of Science, Engineering and Information Technology \\ Federation University Australia \\ Churchill Vic 3842 Australia \\ guojun.lu@federation.edu.au
}

\begin{abstract}
This paper proposes a classifier-free method for extraction of power line wires from aerial point cloud data. It combines the advantages of both grid- and point-based processing of the input data. In addition to the non-ground point cloud data, the input to the proposed method includes the pylon locations, which are automatically extracted by a previous method. The proposed method first counts the number of wires in a span between the two successive pylons using two masks: vertical and horizontal. Then, the initial wire segments are obtained and refined iteratively. Finally, the initial segments are extended on both ends and each individual wire points are modelled as a 3D polynomial curve. Experimental results show both the object-based completeness and correctness are 97\%, while the point-based completeness and correctness are $99 \%$ and $88 \%$, respectively.
\end{abstract}

Index Terms-power line, wire, extraction, modelling, point cloud

\section{INTRODUCTION}

A timely and accurate monitoring of power line corridor features such as pylons, wires (conductors) and vegetation encroachment helps preventing causes for possible unexpected situations, for example, power blackout. At present most of the utility companies largely rely on the manual power line corridor inspection. In such inspections human is engaged in either field trips for in-person physical inspection of the corridor or visual inspection using a large number of aerial images on computers. However, these type of inspections are not only time consuming and cost expensive, but also may be hazardous (e.g., dangerous working condition in field trips). Recently, many of the utility companies are looking for the cost-effective solutions to power line corridor inspection, which could be achieved by exploiting the latest remote sensing technologies like high resolution optical digital cameras and LIDAR (Light Detection and Ranging) system. In fact, the industry now requires 3D models of power line corridors to monitor potentially dangerous cases such as vegetation encroachment and faulty power line components. For example, by using the $3 \mathrm{D}$ power line corridor models the potentially dangerous vegetation can be obtained through estimating possible conductor blowouts according to the simulated wind intensity and growth of trees [1].

This research is funded by Griffith University New Researcher Grant 036.
Compared to other remote sensing data (e.g., multispectral imagery [2]), the airborne LIDAR point cloud data have become a promising data source for 3D modelling such as building rooftop [3] and power line corridor [4] modelling. This is mainly because LIDAR data are now captured cost effectively with high density, high accuracy and multiple echoes of 3D object points. The 3D power line corridor modelling process essentially has two main steps: segmentation of the data into different objects such as pylons, wires and vegetation, and then modelling of the corridor by reconstructing these segmented objects. In our earlier work [5], we have investigated the pylon locations within a power line corridor and achieved 100\% success. This paper presents the subsequent work, the detected pylon locations are exploited in order to facilitate effective and efficient extraction of wires.

There are thousands of kilometers of power lines in any country. Given that power lines can pass through forrest as well as urban areas, the environment is heterogeneous with not only different types of objects like trees, roads and buildings, but also hilly terrain and uncertain weather conditions. The early detection of pylon locations before the extraction of other objects within the corridor helps in two main ways. Firstly, it reduces the amount of data considerably (to process in subsequent steps) by providing a region of interest, i.e., the corridor area. Secondly, the point cloud data between two successive pylons can be processed separately, thus, facilitating for faster processing by using parallel programming.

\section{RELATED WORK}

The methods used for detection, extraction and reconstruction of power lines can be initially divided into two groups: 2D image-based [6] and 3D point-based [4] approaches. The 2D image-based methods use the optical images under the assumption that power lines have uniform brightness, linear property and are parallel to each other. However, these methods are hard to automate and may be unsuitable for 3D modelling. They highly depend on the contrast of the image, i.e., the background and foreground pixel difference in order to detect linear features for power lines. So, they are sensitive to noise and test data types (e.g., urban vs rural). Moreover, due to absence of the height information self-occlusion occurs in the height direction and, therefore, it is difficult to detect 
Fig. 1. Test data set $(3,000 \mathrm{~m} \times 430 \mathrm{~m})$ : (a) input point cloud data and (b) non-ground points. ('N': north direction)

overlapping power lines. In contrast, methods in the 3D pointbased approach first classify different objects within the power line corridor. Then, the 3D modelling of the corridor is based on the several unique properties of the LIDAR data such as accurate height data, multiple echoes and intensity of the returned laser ray.

Depending on how the data is processed the methods in 3D point-based approach can be categorised into two: grid-based [7] and point-based [8] methods. The grid-based methods interpolate the $3 \mathrm{D}$ point cloud data into a $2 \mathrm{D}$ grid space, where each grid cell (pixel) contains representative information such as point height or laser intensity values. They offer an effective way of managing a huge volume of point cloud data and take the advantage of easy application of various low-level computer vision algorithms like segmentation using region growing techniques. However, these methods assume that each grid cell represents only one object, while vertically overlapped multiple objects may exist within the cell. For instance, 3D laser points returned from one of the following pairs of objects may exist in a single grid cell: wire and ground, vegetation and wire, vegetation and building, and wire and pylon. The point-based methods aims to extract (classify) individual objects considering every single point and, thus, they carry out a full investigation of all 3D points to label each point with an object class. Therefore, the benefit of the pointbased methods is that vertically overlapped multiple objects can be labelled with different object classes, although the computational cost of such a full investigation is expensive.

The power line extraction methods in 3D point-based approach are further classified into three types based on how the 
Fig. 2. Outcomes generated by Awrangjeb et al. [5]: (a) Corridors and pylons; and (b) Power line mask. ('N': north direction)

input points are clustered or classified into individual objects in the scene [9]. Methods involving statistical analysis use height, density and number of pulses [10], [11]. Line-based power line extraction methods apply Hough Transform [12] and RANSAC (RANdom SAmple Conseus) [13] algorithms. Supervised classification-based methods first extract several features from the input data and then apply a classification algorithm [8], [9]. Line-based methods are computationally expensive for large data sets and incorrect topographic relationships may be established specially when multiple objects overlap one another vertically. In contrast, the classificationbased methods require large training data sets, which are hard to collect, for desired results and an unbalance sampling in the training set increases the rate of misclassification [10].

This paper proposed a classifier-free 3D point-based power line extraction approach where both the grid-based and pointbased methods are followed for data processing. Therefore, the proposed method combines the advantages of different approaches, while avoids the pitfalls of image-based and classifier-based approaches.

\section{DATA SET}

Fig. 1 shows the test data set from Bindebango, QLD, Australia. Fig. 2 shows the power line corridors and pylons, which were automatically extracted from the test site by the method in [5]. Each power line corridor in Fig. 2 is shown using four consecutive red rectangular segments and within each segment a blue line is shown to indicate the direction of the power line in that segment. In three corridors pylons are shown via circles, squares or diamonds. 
The test site is $3,000 \mathrm{~m}$ in length and $430 \mathrm{~m}$ in width, which varies between $330 \mathrm{~m}$ and $530 \mathrm{~m}$. There are 3 power line corridors and all of them are transmission lines. They have a total of 24 pylons ( 8 on each), which are columns made of steel frames. Note that 10 pylons are shown within each corridor in Fig. 2 including two end points of the corridor. The width of each corridor is about $20 \mathrm{~m}$.

As can be seen in the magnified snapshots in Fig. 1, within each span (i.e., pylon to pylon) Corridor 1 has a total of 14 wires $(6 \times 2+2 \times 1)$ at four height levels $(4+4+4+2)$, while each of Corridors 2 and 3 has 5 wires $(5 \times 1)$ at two height levels $(3+2)$.

The total number of points in the point cloud is $18,500,435$ (see Fig. 1(a)) and the number of non-ground points, shown in Fig. 1(b), is $14,449,675$ (78.10\% of the total points). However, the number of non-ground points within the three corridors is only $3,531,136$ (19.09\% of the total points).

Thus, the aim of the research presented in this paper is to extract points for individual power line wires and, therefore, to effectively model the individual cables. To achieve this objective only the non-ground points within each corridor are processed. Moreover, instead of processing all points within a corridor at a time, only the points between two pylons are processed.

\section{Proposed Wires Extraction Method}

Fig. 3 shows the basic workflow of the proposed wire extraction method. The inputs to the method include the nonground point cloud data $L p_{u p}$ and power line mask $M_{p}$. In order to count the number of wires, two binary masks are used. The first one is a vertical mask across the power line $M_{v}$ that shows the wires on a vertical cross section and helps find the height clusters of wires at different height levels. The second one is $M_{p}$ which helps to count the number of wires horizontally exists on each height cluster. An initial wire segment is then generated for each height cluster. Finally, the initial segment is refined and extended towards both ends and points on each full wire are modelled to generate $3 \mathrm{D}$ wire.

\section{A. Counting Wires in Masks}

The 3D input points $(x, y, z)$ are converted to the 2D coordinate system of $\left(\sqrt{x^{2}+y^{2}}, z\right)$ and a white mask $M_{v}$ is defined with a resolution $r_{s}$. The width of $M_{v}$ is set to the width of the corridor $(20 \mathrm{~m})$ and the height is set to be the minimum and maximum heights of the input points. The value of $r_{s}$ is set to $0.15 \mathrm{~m}$ assuming that the transmission wires that vertically overlap each other have at least $1 \mathrm{~m}$ height difference.

Let $L_{s}$ be the pylon axis, i.e., the line connecting the two successive pylons $P y_{1}$ and $P y_{2}$ and $L_{p}$ be the perpendicular line passing through the midpoint of $L_{s}$ (Fig. 4(a)). The length of $L_{p}$ is set to be the width of the corridor $(20 \mathrm{~m})$. A vertical plane $P l_{v}$ is generated passing through $L_{p}$ such that there are points on both sides of $P l_{v}$ as shown in Fig. 4(b). Starting from the nearest point on any side, the wire points $P_{w}$ within next $1 \mathrm{~m}$ are taken and mapped into an empty $M_{v}$. The wires
Fig. 3. The workflow of the proposed method.

are found as small individual black regions in $M_{v}$ and these are obtained into clusters allowing a neighbourhood of 4 pixels $(0.6 \mathrm{~m})$ between any two black pixels in a cluster. Fig. 4(c) shows eight clusters for $P_{w}$ in $M_{v}$.
Fig. 4. Counting the number of wires: (a) Pylon axis $L_{s}$ and vertical plane $\mathrm{Pl}_{v}$; (b) Input points between an end and a pylon of Corridor 1; (c) Clusters of wires in vertical mask $M_{v}$; and (d) Connected components from pylon mask $M_{p} .\left(v_{c}=\right.$ vertical count of wires and $h_{c}=$ horizontal count of wires. $)$

If a cluster is at most 2 pixels $(0.30 \mathrm{~m})$ high (vertically), then the number of vertical wires $v_{c}=1$ is set for this cluster. 
Otherwise, $v_{c}=2$ which does not happen since there are no vertically close wires in the test scene. Fig. 4(c) shows that for all clusters $v_{c}=1$ is estimated correctly. In order to count the number of horizontal wires (across the power line direction), $P_{w}$ are now mapped into $M_{p}$ and a region of interest (ROI) is defined allowing 2 pixels neighbourhood. The corresponding pixels for this ROI are extracted from $M_{p}$ and a connected component analysis is carried out. Fig. 4(d) shows eight connected components, one for each cluster in Fig. 4(c). For each connected component if the maximum width (or length) is 2 pixels then the number of horizontal wires $h_{c}=1$ is set for this component. Otherwise, $h_{c}=2$ which usually happens for Corridor 1 since there are six pairs of wires and wires in each pair is only about $0.30 \mathrm{~m}$ away from each other. Therefore, these two wires are found into one connected component. The value of $h_{c}$ is mostly estimated correctly. However, sometimes it can be wrong, as shown within the orange coloured ellipse in Fig. 4(d). In $M_{p}$ the single wire at the top left side of the corridor is confused with the double wires below it.

\section{B. Extracting Initial Wire Segments}

Consequently, in order to find the actual values of $v_{c}$ and $h_{c}$ the estimation of $v_{c}$ and $h_{c}$ continues iteratively as above, using $1 \mathrm{~m}$ of wires at a time, for 30 times assuming that the maximum distance between two successive points is $30 \mathrm{~m}$. In fact, for thick wires or for the input data having high point density (e.g., 6 pairs of wires at the bottom of Fig. 4(b)) the number of iterations can be lower; however, for thin wires or for the input data having low point density (e.g., 2 single wires at the top of Fig. 4(b)) the number of iterations can be higher.

At each iteration, all the points $P_{w}$ are marked as 'assigned' and the mean point $P w_{m}$ of $P_{w}$ is stored. Fig. 5(a) shows the input points in cyan dots for 7 wires (in front) at 4 height levels $(3 \times 2+1)$ and the mean points in magenta circles. We have a 4 lists of mean points in this figure. In an iteration, to include a new mean point $P_{n}$ to a list of previously listed mean points, three checks are executed. First, $v_{c}$ and $h_{c}$ values are the same for both the list and $P_{n}$. Second, the line connecting $P_{n}$ and the last mean $P_{l}$ in the list is parallel to the pylon axis $L_{s}$. Third, the height difference between $P_{n}$ and $P_{l}$ is below a threshold, which is calculated based on the distance between these points and the tangent of the maximum possible slope of the wire. For parallelism and height checks, the angle threshold is set as $\theta=22.5^{\circ}$.

\section{Refining Initial Segments}

Some of the wire segments extracted above may be spurious containing only a small number of mean points. So, the segment with only 1 mean point is discarded. Also, some of them may be the resultants of split from a single wire. Therefore, every pair of the candidate segments is further scrutinised. Let $S_{i}=\left\{P_{m, i}\right\}$ and $S_{j}=\left\{P_{n, j}\right\}$, where $m, n \geq 2$, are the set of mean points for two candidate segments $m$ and $n$. Further, let $L_{m}$ and $L_{n}$ are two 3D lines of these two segments. The following check is performed to merge them, if possible. The perpendicular distances from $S_{i}$ to $L_{n}$ are within $1 \mathrm{~m}$ and the height errors between the estimated and actual heights are within $0.5 \mathrm{~m}$. If the check is true, then the longer segment is updated with the information of the shorter segment, which is abandoned thereafter.

After the above merging step, the value of $h_{c}$ is revised based on a majority voting criterion. Let $P_{w}$ be the set of original points (the cyan points in Fig. 5(a)) and $P_{t}$ be the set of the intersection points between the pylon axis $L_{s}$ and the perpendicular lines from $P_{w}$ to $L_{s}$. Points in $P_{w}$ are divided into groups according to the distances of $P_{t}$ from an end point of $L_{s}$ : points within $1 \mathrm{~m}$ distance are in one Group 1, those in between $1 \mathrm{~m}$ and $2 \mathrm{~m}$ distances are in Group 2, and so on. In an iterative procedure, the maximum perpendicular distance difference among the points in each group is obtained and if this is at most $0.3 \mathrm{~m}$ ( 2 pixels), then 1 vote goes for $h_{c}=1$, else 1 vote for $h_{c}=2$. Fig. 5(b) shows one set of single wire (green) and one set of double wires (yellow). For each wire type, two groups of points are shown along $2 \mathrm{~m}$ distance of $L_{s}$. For both cases, the maximum and minimum perpendicular distances are shown in every $1 \mathrm{~m}$ space along $L_{s}$. For the single wire, the difference between the maximum and minimum distances is less than $0.3 \mathrm{~m}$, therefore $h_{c}=1 \mathrm{~m}$ gets 1 vote for each group. In contrast, for the double wires, the difference between the maximum and minimum distances is more than $0.3 \mathrm{~m}$, therefore $h_{c}=2 \mathrm{~m}$ gets 1 vote for each group. After voting for all groups of a wire along $L_{s}$, if $h_{c}=1$ $\mathrm{m}$ gets majority votes, then this wire is decided as a single wire; otherwise, is decided as a double wire.

Fig. 5. Wire extraction: (a) Initial segments; (b) Examining single and double wires; (c) Extending an initial segment; and (d) 3D models for the sample scene in Fig. 4(b).

\section{Extracting Final Wires}

Once initial wire segments are refined as above, they are now extended according to the number of mean points $P w_{m}$. In order to avoid spurious wire extension, wires with the largest number of $P w_{m}$ is extended first, then the second line 
with the largest number of $P w_{m}$, and so on. For each initial segment, it is extended on both sides iteratively, $1 \mathrm{~m}$ at a time. On each side, a 3D line $L_{3}$ is constructed using the last 5 mean points (pink points in Fig. 5(a)) in that side of the wire and only unassigned input points within $1 \mathrm{~m}$ neighbourhood of $L_{3}$ are considered to extend the side. Both $L_{3}$ and the unassigned points are dynamically updated after every 5 iterations. Note that for the thin wire where we do not have many input points, minimum 2 mean points are still required to construct $L_{3}$.

Fig. 5(c) shows the 'unassigned' points $P_{u}=\left(x_{u}, y_{u}, z_{u}\right)$ in black circles. In each iteration, heights $z_{u}$ of $P_{u}$ are estimated as $z_{e}$ with respect to $L_{3}$ by considering the intersection points $P_{t}$ between $L_{3}$ and the perpendicular lines from $P_{u}$ to $L_{3}$. The height error thresholds $\xi_{T}$ are dynamically estimated by considering the maximum slope of wire in this segment being extended to be $\theta$ and the distances $d_{t}$ from the nearest end of $L_{3}$ to $P_{t}: \xi_{T}=d_{t} \tan (\theta)$ (see Fig. 5(c)). The end points of $L_{3}$ is decided by the last added assigned point to the wire. For an unassigned point $P_{u}$, it is assigned to the wire if its height error $\left|z_{u}-z_{e}\right|$ is less than $\xi$. For all newly assigned points in an iteration, their mean is added to the wire segment. Fig. 5 (c) shows the newly added mean points in orange circles.

After extraction of a wire, if it is a double wire $\left(h_{c}=2\right)$, its mean points are used to divide all its 'assigned' points into two parts. As is evident from the bottom three pairs of wires in Fig. 5(a), the mean (magenta) points can be used to separate the (cyan) points for two wires. Otherwise, if it is a single wire $\left(h_{c}=1\right)$, it has only one part, as can be evident from the top single wire in Fig. 5(a). Finally, each part of a wire is modelled as a 3 D polynomial curve $\left(z=a\left(x^{2}+y^{2}\right)+b * \sqrt{x^{2}+y^{2}}+c\right)$ using MATLAB polyfit function. Fig. 5(d) shows the 3D wires for the input point cloud in Fig. 4(b). Six pairs of double wires are shown in green and magenta colours, while two single wires are shown in cyan colour.

\section{E. Results and Discussions}

Fig. 6 shows the wire models for the first 3 spans of all three corridors. For Corridor 1, all 14 wires were correctly extracted and modelled in the first 2 spans; however, for the third span the top two single wires were extracted as doubles because of the noisy input points. As shown in Fig. 7(a) within the top ellipse, clearly there are two rows of wire points captured for the single wire, which is why they were extracted and modelled as a pair of wires. The same noisy data trend was observed for all double wires, e.g., within the double wires within the bottom ellipse, where there are four rows of data for two actual wires. Since, the proposed method is only capable of obtaining double wires, it did not extract 4 wires here. Fig. 7(b) shows the case where two top single wires are extracted correctly despite shortage of the input points (i.e., where initial wire segments are found). However, in the case of extreme shortage of points (i.e., where no wire segments are found), the proposed algorithm cannot extract the wire at all (see Fig. $7(\mathrm{c})$ ).

In order to evaluate the objective performance, the ground truth data were collected manually. In terms of object-based evaluation (counting the number of wires), completeness, correctness and quality values are $97.22 \%, 97.22 \%$ and $94.6 \%$. In terms of point-based accuracy (counting the number of points), completeness, correctness and quality values are $88.18 \%$, $99.95 \%$ and $88.15 \%$. The low point-based completeness was mainly due to missing of some wire points which were confused with either as pylons or trees.

\section{CONCLUSION}

We have presented a classifier-free method for extraction of power line wires from aerial point cloud data. By using two masks (vertical and horizontal), we first count the number of wires between two successive pylons. Then, we follow an iterative procedure to obtain the initial wire segments, which are refined and extended on both ends. Finally, extracted wire points on an individual wire are modelled as a 3D polynomial curve. Experimental results show both the object-based and point-based completeness and correctness are high. The future work includes the extraction of power line wires even in noisy input data and analyse the performance of the 3D wire models.

\section{REFERENCES}

[1] Y. Jwa, G. Sohn, and H. B. Kim, "Automatic 3d powerline reconstruction using airborne lidar data," International Archives of the Photogrammetry, Remote Sensing and Spatial Information Sciences, vol. XXXVIII, no. 3/W8, pp. 105-110, 2009.

[2] M. Awrangjeb, C. Zhang, and C. S. Fraser, "Automatic extraction of building roofs using LIDAR data and multispectral imagery," ISPRS Journal of Photogrammetry and Remote Sensing, vol. 83, no. 9, pp. 1-18, 2013.

[3] M. Awrangjeb and C. S. Fraser, "Automatic segmentation of raw LIDAR data for extraction of building roofs," Remote Sensing, vol. 6, no. 5, pp. 3716-3751, 2014

[4] Y. Jwa and G. Sohn, "A piecewise catenary curve model growing for 3d power line rensostruction," Photogrammetric Engineering \& Remote Sensing, vol. 78, no. 12, pp. 1227-1240, 2012.

[5] M. Awrangjeb, D. Jonas, and J. Zhou, "An automatic technique for power line pylon detection from point cloud data," in Proc. Digital Image Computing: Techniques and Applications, Sydney, Australia, 29 Nov-1 Dec 2017

[6] Y. Chen, Y. Li, H. Zhang, L. Tong, Y. Cao, and Z. Xue, "Automatic power line extraction from high resolution remote sensing imagery based on an improved radon transform," Pattern Recognition, vol. 49, pp. 174186, 2016.

[7] S. Clode and F. Rottensteiner, "Classification of trees and powerlines from medium resolution airborne laserscanner data in urban environments," in Proc. APRS Workshop on Digital Image Computing, Brisbane, Australia, 2005, p. CDROM.

[8] H. B. Kim and G. Sohn, "Point-based classification of power line corridor scene using random forests," Photogrammetric Engineering \& Remote Sensing, vol. 79, no. 9, pp. 821-833, 2013.

[9] Y. Wang, Q. Chen, L. Liu, D. Zheng, C. Li, and K. Li, "Supervised classification of power lines from airborne lidar data in urban areas," Remote Sensing, vol. 9, no. 8, pp. 771-786, 2017.

[10] L. Zhu and J. Hyyppä, "Fully-automated power line extraction from airborne laser scanning point clouds in forest areas," Remote Sensing, vol. 6 , no. 11 , pp. $11267-11282,2014$.

[11] M. Ritter and W. Benger, "Reconstructing power cables from lidar data using eigenvector streamlines of the point distribution tensor field," in Proc. International Conference in Central Europe on Computer Graphics, Visualization and Computer Vision, Plzen, Czech Republic, 2012, pp. 223-230.

[12] D. Grigillo, S. Ozvaldic, A. Vrecko, and M. K. Fras, "Extraction of power lines from airborne and terrestrial laser scanning data using the hough transform," Geodetski Vestnik, vol. 59, no. 2, pp. 246-261, 2015.

[13] T. Melzer and C. Briese, "Extraction and modeling of power lines from als point clouds," in Proc. Workshop of the Austrian Association for Pattern Recognition, Hagenberg, Austria, 2004, pp. 47-54. 
Fig. 6. Wire models for first 3 spans from the test scene. Data in the dashed rectangles are zoomed in Figs. 7(a)-(c). Note that the pylons are artificially inserted for visualisation only.

Fig. 7. Some difficult cases in the test scene: (a) Noisy input points; (b) Shortage of input points on thin wires; and (c) absence of input points on thin wires. 